\title{
Silicone Implant-Based Paranasal Augmentation for Mild Midface Concavity
}

\author{
Joo Hyun Kim, \\ Min Su Jung, \\ Byeong Ho Lee, \\ Hii Sun Jeong, \\ In Suck Suh, \\ Duk Kyun Ahn \\ Department of Plastic and Reconstructive \\ Surgery, Kangnam Sacred Heart Hospital, \\ Hallym University Medical Center, Seoul, \\ Korea
}

No potential conflict of interest relevant to this article was reported.

\begin{abstract}
Background: Midface concavity is a relatively common facial feature in East Asian populations. Paranasal augmentation is becoming an increasingly popular procedure for patients with mild concavity and normal occlusion. In this study, we evaluate clinical outcomes following a series of paranasal augmentation.

Methods: A retrospective review was performed for patients with Class I occlusion who had undergone bilateral paranasal augmentation using custom-made silicone implants, between October 2005 and September 2013. Patient charts were reviewed for demographic information, concomitant operations, and postoperative complications. Preoperative and postoperative (1-month) photographs were used to evaluate operative outcome. Results: The review identified a total of 93 patients meeting study criteria. Overall, aesthetic outcomes were satisfactory. Five-millimeter thick silicone implant was used in 81 cases, and the mean augmentation was $4.26 \mathrm{~mm}$ for this thickness. Among the 93 patients, 2 patients required immediate implant removal due to discomfort. An additional 3 patients experienced implant migration without any extrusion. Nine patients complained of transient paresthesia, which had resolved by 2 weeks. There were no cases of hematoma or infection. All patients reported improvement in their lateral profile and were pleased at follow-up. Complications that arose postoperatively included 9 cases of numbness in the upper lip and 3 cases of implant migration. All cases yielded satisfactory results without persisting complications. Sensations were fully restored postoperatively after 1 to 2 weeks.

Conclusion: Paranasal augmentation with custom-made silicone implants is a simple, safe, and inexpensive method that can readily improve the lateral profile of a patient with normal occlusion. When combined with other aesthetic procedures, paranasal augmentation can synergistically improve outcome and lead to greater patient satisfaction.
\end{abstract}

Keywords: Piriform cortex / Silicones / Prosthesis and Implants

\section{INTRODUCTION}

By the very nature of its centrality, the midface is an important area for determining the overall outline and impression of a face. Hence, midface concavity can be extremely conspicuous and aes-

Correspondence: Duk Kyun Ahn

Department of Plastic and Reconstructive Surgery, KangNam Sacred Heart Hospital, Hallym University College of Medicine, 1 Singil-ro, Yeongdeungpo-gu, Seoul 07441, Korea

E-mail: drahn@hallym.or.kr

Received April 23, 2015 / Revised August 23, 2015 / Accepted December 3, 2015 thetically displeasing. Public interest in appearance and beauty is increasing, and the number of patients who wish to improve midface concavity is also on the rise.

When a concavity is accompanied by occlusal disharmony, various skeletal operations are used. However, skeletal surgery has disadvantages, such as the need for perioperative orthodontic treatment, general anesthesia-associated morbidity, and increased costs. With patient demand increasing for minimally-invasive treatments, paranasal augmentation is gradually rising as an alternative to skeletal surgery in Korea. 
In this study, we explored the potential advantages of performing paranasal augmentation with silicone implants in patients with mild midface concavity.

\section{METHODS}

Upon Institutional Review Board exemption, this retrospective review was performed for patients with Class I occlusion who had undergone bilateral paranasal augmentation using custom-made silicone implants, between October 2005 and September 2013. Patient charts were reviewed for demographic information, concomitant operations, and postoperative complications. Preoperative and postoperative (1-month) photographs were used to evaluate operative outcome.

\section{The surgical method}

Before each operation, patients were given a gargling solution of betadine and saline mixture. All operations were performed with patients under local anesthesia and monitored anesthesia care. The upper gingivobuccal sulcus incision (1.5-2 cm long) was made just lateral to the pyriform aperture. The dissection was carried in the subperiosteal plane to create ample space for implants to be inserted.

Most implants used to augment the midface were custommade silicone implants (WooRhi Medical Group, Seoul, Korea), and were carved to meet specific needs of each patient (Fig. 1). Different sizes of implants were used to optimally fit the concavity, which reduced the need for implant fixation.

With the periosteal dissection complete, the implants were introduced into the pocket and positioned to sit flush to the bone. If implant migration was a possibility, the implant was secured to the nasal process of the maxilla. A hole was drilled at the margin of the lower maxilla, and the implant was fixed to the bone with a 4-0 nylon suture.

The sulcus incision was closed with 4-0 vicryl suture. Compressive tape dressing was applied to prevent swelling and hematoma formation. Postoperative regimen included prophylactic antibiotics and gargling. Compressive dressing was maintained for 5 days, and stitches were removed from 8 to 10 days after operation.

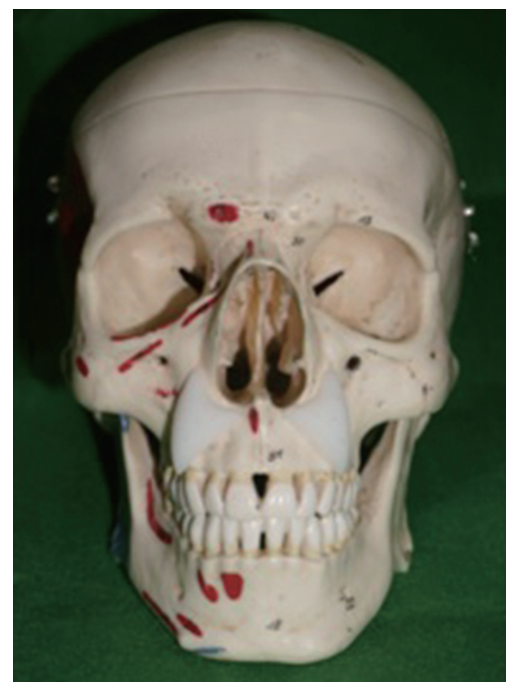

Fig. 1. The silicone implants are designed to augment both paranasal areas.

\section{RESULTS}

The review identified a total of 93 patients meeting study criteria. The mean age was 26 years (range, 19 to 48 years). More female patients underwent paranasal augmentation than male patients did (85 females to 8 males). Paranasal augmentation was performed as the only procedure in 20 of the patients. The most commonly performed concomitant procedure was rhinoplasty (53), followed by genioplasty (15) and angle resection (5).

The thickness of the silicone implants ranged from 3 to $6 \mathrm{~mm}$, depending on the degree of midface concavity. The most commonly used implant thickness was $5 \mathrm{~mm}$. Patient follow-up ranged from 8 to 60 months with a mean of 30 months.

Overall, aesthetic outcomes were satisfactory. The nasolabial folds were compared between the preoperative and 1-month photographs to evaluate augmentation of the soft tissue in the area. More specifically, the degree of augmentation was evaluated by the distance between the tragus to the nasolabial fold on the lateral profile. Five-millimeter thick silicone implant was used in 81 cases, and the mean augmentation was $4.26 \mathrm{~mm}$ for this thickness. In the 4 patients receiving 3-mm implants, nasal folds were augmented by $2.48 \mathrm{~mm}$. Four-millimeter implants were used in 6 cases, with augmentation of $3.75 \mathrm{~mm}$. In two cases, 6-mm implants were used, with augmentation of $5.2 \mathrm{~mm}$ (Table 1). 
Postoperative edema subsided within the first five days. Among the 93 patients, 2 patients required immeidate implant removal due to discomfort. An additional 3 patients experienced implant migration without any extrusion. Nine patients complained of transient paresthesia, which had resolved by 2 weeks. There were no cases of hematoma or infection.

\section{Case 1}

This 35-year-old woman underwent paranasal augmentation with a 5-mm thick implant. Postoperative photograph reveals improved midface profile, with considerable augmentation of the paranasal region (Fig. 2).

\section{Case 2}

This 24-year-old woman presented with "protruding mouth" and Class I occlusion. She underwent paranasal augmentation with

Table 1. Paranasal augmentation on lateral profile

\begin{tabular}{|c|c|c|}
\hline \multirow{2}{*}{$\begin{array}{l}\text { Thickness } \\
\text { of silicone }\end{array}$} & \multirow{2}{*}{$\begin{array}{l}\text { No. of } \\
\text { usage }\end{array}$} & Soft tissue augmentation (nasolabial fold) \\
\hline & & Distance from tragus to nasolabial fold \\
\hline $3 \mathrm{~mm}$ & 4 & $2.48 \mathrm{~mm}$ \\
\hline $4 \mathrm{~mm}$ & 6 & $3.75 \mathrm{~mm}$ \\
\hline $5 \mathrm{~mm}$ & 81 & $4.26 \mathrm{~mm}$ \\
\hline $6 \mathrm{~mm}$ & 2 & $5.2 \mathrm{~mm}$ \\
\hline
\end{tabular}

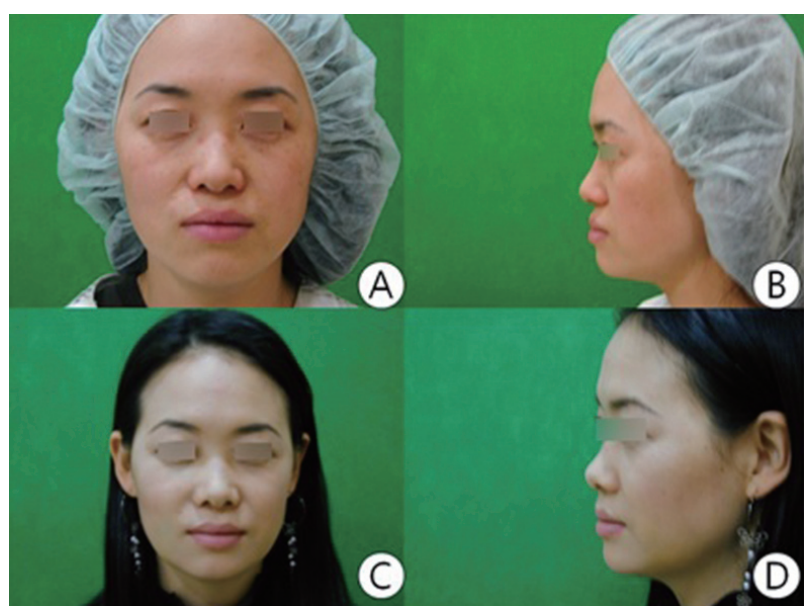

Fig. 2. A 35-year-old woman with lower midface retrusion and neutroocclusion underwent paranasal augmentation. Preoperative (A, B) and postoperative $(C, D)$ photographs. 4-mm implant and aesthetic rhinoplasty (Fig. 3).

\section{Case 3}

This 25-year-old woman wished for a significant improvements in the lateral facial contour. She underwent multiple procedures, including forehead augmentation, genioplasty, rhinoplasty, and paranasal augmentation with a 5-mm implant (Fig. 4).

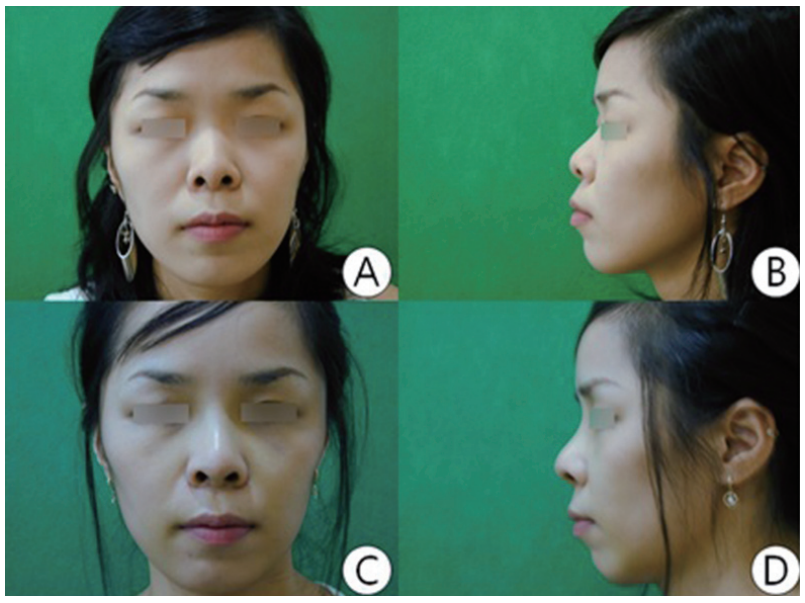

Fig. 3. This 24-year-old woman underwent a combination of open rhinoplasty with tip graft and paranasal augmentation. The augmentation led to an increase in the lower midface projection, and the effect of rhinoplasty is more pronounced. Preoperative (A, B) and postoperative $(C, D)$ photographs.

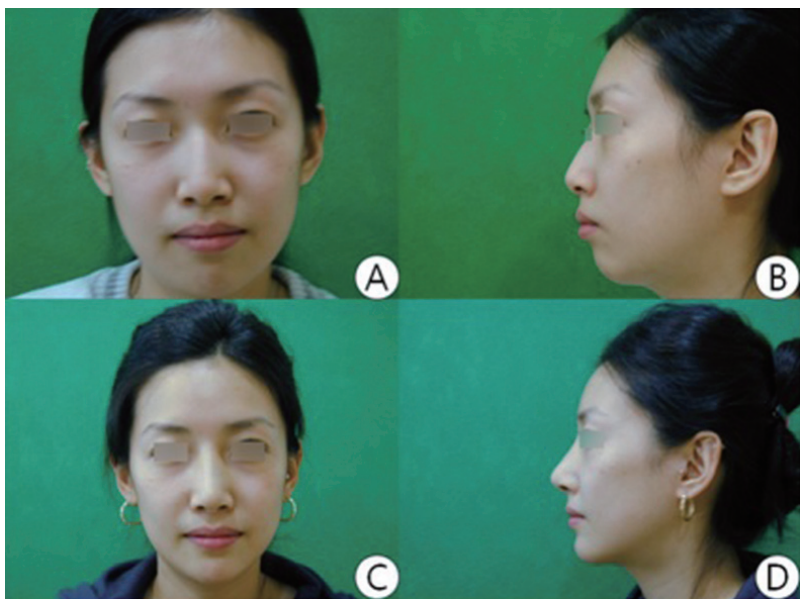

Fig. 4. A 25-year-old woman with a flat forehead, nasal hump, and chin retrusion underwent forehead augmentation, genioplasty, rhinoplasty, and paranasal augmentation. Preoperative (A, B) and postoperative $(\mathrm{C}, \mathrm{D})$ photographs. 


\section{DISCUSSION}

Midface concavity is a common aesthetic complaint among Asian patients. Convexity of the midface builds up a youthful impression, whereas concavity is associated with downtrodden and aged appearance. As the concept of beauty continued to evolve, the desire for addressing midface concavity has expanded and led the development of materials and methods to augment the paranasal region.

Midface concavities has three categories of etiology: congenital, developmental, and acquired [1]. Congenital causes include deficiencies related to facial clefts (e.g., Binder's syndrome) and midface hypoplasia. A deficiency in lower midface projection is common in patients with surgically corrected clefts. Both alterations in soft tissue and scarring are believed to restrict growth of maxilla and palatine after cleft repair [2]. Race is also a congenital factor associated with midface concavity, which are more common in populations of Asian and African descent [3].

The primary developmental cause of midface concavity is tooth extraction, which can result in underdevelopment of the skeleton surrounding the bicuspids, which in turn causes flattening of the maxillary platform. Acquired causes consist mainly of trauma and may reflect the absence or malposition of the maxillary skeleton [2].

Severe retrusion of the midface requires skeletal operations, such as Le Fort osteotomy, but minor concavity can be corrected by implant-based augmentation of the paranasal region. Paranasal augmentation requires a relatively shorter operation compared with osteotomy-based solution, and may be performed under local anesthesia. The duration of admission and recovery is short due to the technical easiness of the surgery.

This procedure changes the lateral face in a minimally invasive manner, correcting only the contour of the profile without any osteotomies. The implant-based augmentation in the paranasal area allows the nasal tip to look more natural. In the present study, we evaluated the outcomes of paranasal implantation using preoperative and postoperative photographs. In our patients, 5-mm thick implants were associated with $4.26 \mathrm{~mm}$ augmentation in the paranasal area. In a study by You et al. [4], the use of $4.5 \mathrm{~mm}$ Medpor implants were associated with a paranasal augmentation of $3.27 \pm 1.16 \mathrm{~mm}$.
Several limitations exist in our study. Surgical outcomes were evaluated using clinical photographs, and the degree of augmentation was not measured directly on patients themselves. Also, the outcomes could not be considered as the sole result of implantbased augmentation, as the interventions varied between patients who received augmentation alone and those who received concurrent operations. Such variations could introduce changes to lateral profile. Nevertheless, this procedure appears to be a convenient method of improving midface concavity.

Paranasal augmentation can be performed using a variety of implant materials, including autografts and allografts [5]. Autograft materials such as the iliac bone, cranium, and the coronoid process have been reported for paranasal augmentation. Bone grafts have the necessary strength, with a low risk for rejection and infection. In general, autografts are optimal for patients who do not wish for implantation of a foreign body. However, autografts have two major drawbacks, which are donor-site morbidity and graft resorption. Iliac bone grafts are associated with a higher resorption rate, and patients often suffer from severe pain at the donor site, making ambulation more difficult in the early post-operative period. In comparison, resorption rate is lower for the cranial bone because it contains a higher proportion of cortical bone. Donor-site morbidities can be serious for cranial bone grafts, such as intracerebral bleeding, cerebrospinal fluid leakage, and perforation of the sagittal sinus-with reports of fatal outcomes.

Allografts offer many advantages over autografts. The use of alloplastic materials shortens operation time, and implant materials are readily accessible. No donor-site morbidities can exist for using an allograft [6]. Allograft materials used for paranasal augmentation include Proplast (Teflon interwoven with organic fibers, Vitek, Houston, TX, USA), hydroxyapatite, Gore-Tex, Medpor (Porex Surgical, Newnan, GA, USA), and silicone. Among these, Medpor and silicone are more widely used for this application.

Porous polyethylene mesh, Medpor, has the advantage of being a non-antigenic, non-resorptive, and highly stable material. It does not erode through overlaying thin soft tissues and may be used in many sites without loading. Additionally, the porous structure facilitates osseous ingrowth and fibrovascular infiltration, which enhances implant stabilization and increases resistance to infec- 
tion $[7,8]$. Compared with autograft materials; however, Medpor still is at a higher risk for exposure and inflammation [9].

Despite the advantages of using Medpor, we chose silicone implants for paranasal augmentation at our institution for several reasons. First, silicone implants have a smooth surface and are more flexible. These properties allow the implants to be easily inserted through relatively smaller incisions, which help the implants fit in surgical pockets. However, the length of incisions may be different depending on experience or technique of the operating surgeon. In the study, the implants were inserted through incisions which were 1.5 to $2 \mathrm{~cm}$ in length. Second, sculpting is easier for silicone implants than for porous implants, which helps in achieving the desired shape and symmetry of the midface. Preparing Medpor for implantation is inconvenient because the implant must to be made pliable by placing it in warm saline before molding and carving it to the necessary size and shape. Third, silicone is cost-effective. Furthermore, there is no need to fix silicone with screws.

One disadvantage of using silicone implant is the migration from bone resorption caused by insufficient vasluarization, which we have observed in 3 out of the 93 patients. Migrated cases were undergone immediate removal and reinsertion of the implant. When implants were re-inserted, the chance of migration was reduced by fixing the implant to the nasal process of the maxilla. In addition, proper incision size is very important for reducing migration rate and before closing the buccal incision, we concurrently sutured the silicone implants with periosteum. Finally, the rates of infection with a silicone implant were equal to or lower than that of porous material. Peled et al. [10] reported that silicone and Medpor have similar risks for infection. Silicone implants, when placed into appropriately sized pockets and held securely by surrounding tissues, are encapsulated by fibrous tissue with a minimal inflammatory response $[11,12]$. In our study, none of the patients experienced infection of the implanted pocket, nor did they experience capsular contracture around the silicone implant.

In summary, paranasal augmentation with silicone implants appears to be an excellent surgical method for correcting midfacial concavities with normal occlusion in the Korean population.

Midface concavity is a relatively common defect in the Asian population. Paranasal augmentation may be a useful substitute for orthognathic surgical procedures if occlusions are normal or have been corrected. This procedure can produce a subtle but noticeable aesthetic improvement, thus creating a softer and more youthful facial expression

Paranasal augmentation can be easily performed alone or together with other aesthetic operations. This procedure has good synergy with operations such as rhinoplasty, genioplasty, and angle resection. In this study, we have used silicone implants for ease of use, cost effectiveness, and flexibility. Results were satisfactory without severe or persistent complications. In conclusion, implant-based paranasal augmentation can improve facial profile of patients with minimal midface deficiency and normal occlusions.

\section{REFERENCES}

1. Ohjiya Y, Sakamoto Y, Kamiishi H. Our principle for protrusion of the mandibular angle. Jpn Soc Aesthet Plast Surg 1988;10:44.

2. Park H, Chun KW, Kye MS, Dhong ES, Yoon ES. Midface augmentation using bony segments obtained from sagittal splitting angle ostectomy in asians. Plast Reconstr Surg 2008;121:578-86.

3. Yaremchuk MJ, Israeli D. Paranasal implants for correction of midface concavity. Plast Reconstr Surg 1998;102:1676-84.

4. You JS, Kim SG, Oh JS, Lim KS, Shin SM, Kim CM. Paranasal augmentation using medpor implant and changes in lateral profile. Oral Biol Res 2013;7:125-30

5. Fanous N, Yoskovitch A. Premaxillary augmentation: adjunct to rhinoplasty. Plast Reconstr Surg 2000;106:707-12.

6. Choung PH, Kim SG. The coronoid process for paranasal augmentation in the correction of midfacial concavity. Oral Surg Oral Med Oral Pathol Oral Radiol Endod 2001;91:28-33.

7. Romano JJ, IliffNT, Manson PN. Use of Medpor porous polyethylene implants in 140 patients with facial fractures. JCraniofac Surg 1993;4:142-7.

8. Atherton D, Haers P. Midfacial augmentation in teenage cleft patients using malar and paranasal Medpor implants. Int J Oral Maxillofac Surg 2014;43:824-6.

9. Kim SK, Yoon CM, Kim MH, Kim MS, Lee KC. Considerations for the management of cryptotia based on the experience of 34 patients. Arch Plast Surg 2012;39:601-5.

10. Peled ZM, Warren AG, Johnston P, Yaremchuk MJ. The use of alloplastic materials in rhinoplasty surgery: a meta-analysis. Plast Reconstr Surg 2008;121:85e-92e.

11. Sclafani AP, Romo T 3rd. Biology and chemistry of facial implants. Facial Plast Surg 2000;16:3-6.

12. de la Pena-Salcedo JA, Soto-Miranda MA, Lopez-Salguero JF. Intranasal surgical approach for malar alloplastic augmentation. Aesthet Surg J 2012;32:27-38. 